This is a preprint of: D.Z.Wang, R.A. Dorey, Formation of PZT Thick Film Single Elements using EHDA Deposition, Materials Science Forum, 628-629, 405-410, 2009

Please quote the above paper when referencing this work

\title{
Formation of PZT thick film single elements using EHDA deposition
}

\author{
D. Z. Wang ${ }^{1,2, a *}$ and R. A. Dorey ${ }^{3, b}$ \\ ${ }^{1}$ Key Laboratory for Micro/Nano Technology and System of Liaoning Province, Dalian University of \\ Technology, Dalian, Liaoning Province, CHINA \\ ${ }^{2}$ Key Laboratory for Precision and Non-traditional Machining Technology of Ministry of Education, \\ Dalian University of Technology, Dalian, Liaoning Province, CHINA \\ ${ }^{3}$ Microsystems \& Nanotechnology Centre, Cranfield University, Bedfordshire MK43 0AL, UK \\ ad.wang@dlut.edu.cn, ${ }^{b}$ r.a.dorey@cranfield.ac.uk
}

Keywords: PZT, Micromoulding, Electrohydrodynamic atomization, MEMS

\begin{abstract}
In this paper, electrohydrodynamic atomization combined with a polymeric micromoulding technique was used to form PZT single element devices using a PZT sol-gel slurry without an etching process. The PZT single element device was initially designed to work as a piezoelectric ultrasonic transducer consisting of a circular or a square of various sizes, which was produced and used to evaluate the process. The resulting PZT device had a homogenous microstructure. It was observed that the relative permittivity of the circular and square single element devices was especially high at small size due to the fringe effect. The results show that the radius and width of the PZT single circular and square element devices with a thickness of $15 \mu \mathrm{m}$ should be bigger than $400 \mu \mathrm{m}$ in order to reduce the fringe effect.
\end{abstract}

\section{Introduction}

Piezoelectric thick films show great potential for micro-electromechanical systems (MEMS) application such as ultrasonic transducers working in thickness mode. Ultrasonic transducers are widely used today especially in the field of medical imaging systems. The continuous demand of high-resolution images requires desirable materials, fabrication techniques and design of structures in generation of ultrasonic transducer. Lead zirconate titanate (PZT) is a suitable material for ultrasonic transducers because of its high piezoelectric constant, relative permittivity and electromechanical coupling factor [1]. High frequency in the region of $30-70 \mathrm{MHz}$ also needs to be obtained for getting high resolution images [2]. Frequency is in inverse with of PZT film thickness. Therefore, thicknesses of $10-100 \mu \mathrm{m}$ need to be achieved to meet the high frequency requirement, which represents a significant technical challenge using thin film fabrication methods such as physical vapour deposition and chemical vapour deposition, due to the slow deposition rates and high levels of stress generated during processing which can lead to crack of the film [4]. The use of conventional bulk ceramic processing with subsequent machining and bonding is wasteful of material and time consuming [4]. Other thick film fabrication techniques based on sintering of ceramic particles such as screen printing are limited by high temperature processing which can easily result in damage to the bottom electrode and substrate [5].

Electrohydrodynamic atomization (EHDA) is a physical process where an electrified liquid is dispersed to fine droplets due to an electrostatic force working on the charged surface of a liquid. The 
electrical shear stress elongates the liquid meniscus formed at the outlet of a capillary, to the form of a cone and/or a jet which next deforms and disrupts into droplets as a result of the electric and mechanical force [6]. This makes it possible to produce fine droplets using a coarse nozzle with a dependence on electrohydrodynamic force rather than the nozzle size. The use of suspensions in EHDA has shown great potential in recent years and has been introduced to form microstructures in bioengineering [7] and chemical engineering [8]. Ink-jet printing (IJP) is commonly used as a deposition method to form ceramic structures [9]. Compared with IJP, EHDA offers two distinct advantages with the use of suspension: a) the size of deposition product is independent of nozzle diameter and $b$ )there is less risk of nozzle blockage.

In this work, a PZT composite sol-gel slurry was prepared, which helped to produce PZT films of a wide range of thickness with low sintering temperature [10]. For the purpose of this work, EHDA technique combined with polymeric micromoulding process was employed to deposit PZT single elements from the PZT composite sol-gel slurry.

\section{Experimental details}

Mask design. Three 5" Chromium/glass photomasks were prepared. One was for bottom electrode deposition, another one was for PZT deposition, and the third one was for top electrode deposition. Two types of PZT thick film element features were designed on the masks. One type of features was a series of circular elements with radius varying from $70 \mu \mathrm{m}$ to $1000 \mu \mathrm{m}$; another type of features was a series of square elements with width varying from $50 \mu \mathrm{m}$ to $1500 \mu \mathrm{m}$. Within these circular and square elements there were two types of top electrodes: one type had extension pad, and the other type had no extension pad. Using these test features, properties of the PZT thick film single element devices in terms of electrode feature and size were examined.

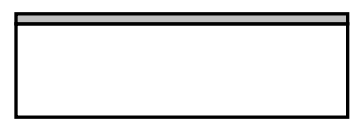

(1) Spin coating of AZ9260 Photoresist

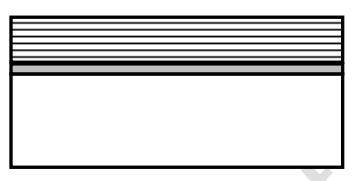

(2)Pattern of photoreisist unver UV light

Fig. 1, Flow chart illustrating the PZT thick film element fabrication route using EHDA deposition

(3) EHDA deposition of PZT structure sinter PZT structure
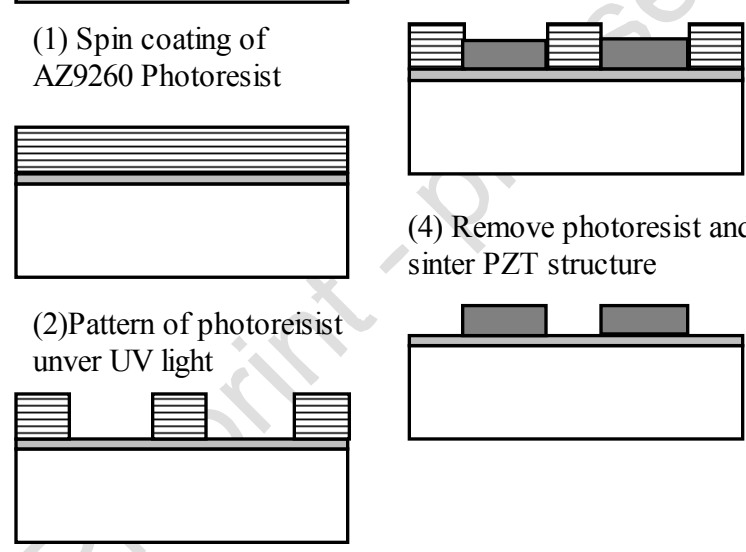

(4) Remove photoresist and

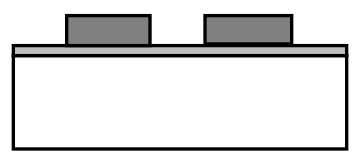

Polymeric micromould formation. The fabrication route of PZT single element is shown in Figure 1, which is photolithography coupled with an EHDA deposition technique. Prior to any deposition the Si wafer was cleaned using acetone/propan-1-ol on a spinner and then dried at $100{ }^{\circ} \mathrm{C}$ for 3 minutes. The top and bottom electrode were deposited with a layer of Ti/Pt $(8 / 100 \mathrm{~nm})$ using RF magnetron
Table 1, Composition of the PZT slurry using in the EHDA deposition process

\begin{tabular}{|l|l|}
\hline PZT powder & $10 \mathrm{~g}$ \\
\hline PZT sol & $14.2 \mathrm{ml}$ \\
\hline Sintering aid $\mathrm{Cu}_{2} \mathrm{O} / \mathrm{PbO}$ & $0.069 / 0.428 \mathrm{~g}$ \\
\hline Dispersant $\mathrm{KR} 55$ & $0.2 \mathrm{~g}$ \\
\hline Zirconia ball-milling media & $100 \mathrm{~g}$ \\
\hline
\end{tabular}


sputtering (Nordiko Ltd., Hampshire, UK). The deposition features were prepared by spin coating a layer of AZ1818 photoresist and dried at $115^{\circ} \mathrm{C}$ for $90 \mathrm{~s}$, then exposed under UV light for $10 \mathrm{~s}$ and revealed in the developer (MF-319) for $40 \mathrm{~s}$. Three layers of AZ9260 positive photoresist (MicroChemicals GmbH, Ulm, German) were spun onto a 4" silicon wafer to achieve a polymer film approximately $38 \mu \mathrm{m}$ thick as polymeric micromould for the EHDA deposition of PZT structure. The preparing procedures are as follows: the first layer of photoresist was spun down at $2400 \mathrm{rpm}$ for $60 \mathrm{~s}$, and then baked on a hot plate at $100^{\circ} \mathrm{C}$ for $160 \mathrm{~s}$; the second and third layers were spun down at 2100 rpm for $60 \mathrm{~s}$, and then baked at $110^{\circ} \mathrm{C}$ for $160 \mathrm{~s}$. The spin coated photoresist was then exposed to UV light on a MA56 mask aligner (SUSS MicroTec. Lithography GmbH, Garching, Germany) at a beam intensity of $6.2 \mathrm{mWcm}^{-2}$ for $403 \mathrm{~s}$. The polymer micromould structures were developed in the solution of diluted AZ400K (1:4 in water) for approximately 8 minutes to obtain the micromould features. After being cleaned with distilled water the micromoulds were dried at $100{ }^{\circ} \mathrm{C}$ for $120 \mathrm{~s}$ before the EHDA deposition of PZT films.

EHDA deposition of PZT. The PZT composite sol-gel slurry was prepared from PZT powder (PZ 26, Ferroperm, Denmark) with a mean particle size of $\sim 0.6 \mu \mathrm{m}$, PZT sol, dispersant KR 55 (Ken-React Lica 38, KenRich) and $\mathrm{Cu}_{2} \mathrm{O} / \mathrm{PbO}$ sintering aid. The composition of the PZT slurry is shown in Table 1. The dispersant was added to stabilize the slurry. The sintering aid helps to increase the density and piezoelectric properties of the sintered PZT film. All these components were mixed in a nitrogen environment and then ball-milled on a roller for $24 \mathrm{~h}$. The PZT sol was prepared from the precursors lead acetate, titanium isopropoxide and zirconium propoxide. $3.55 \mathrm{~g}$ of titanium (IV) isopropoxide (99.99 wt.\% purity) was added to $5.39 \mathrm{~g}$ of zirconium (IV) propoxide (76 wt.\% in 1-propanol) prior to the addition of the solvents 1-propanol (99.7 wt.\% purity, $5 \mathrm{ml}$ ) and glacial acetic acid (99.8 wt.\% purity, $10 \mathrm{ml})$. An excess (9.95 g) of lead (II) acetate trihydrate was then added to the solution and the system was refluxed at a low heat for 30 minutes. The sol concentration was adjusted to $0.42 \mathrm{M}$ by adding $13.2 \mathrm{ml}$ of 1-propanol and $12 \mathrm{ml}$ of acetic acid. The chemical stoichiometric ratio of the metal ions in the PZT sol was Pb 1.10: Zr 0.48: Ti 0.52.

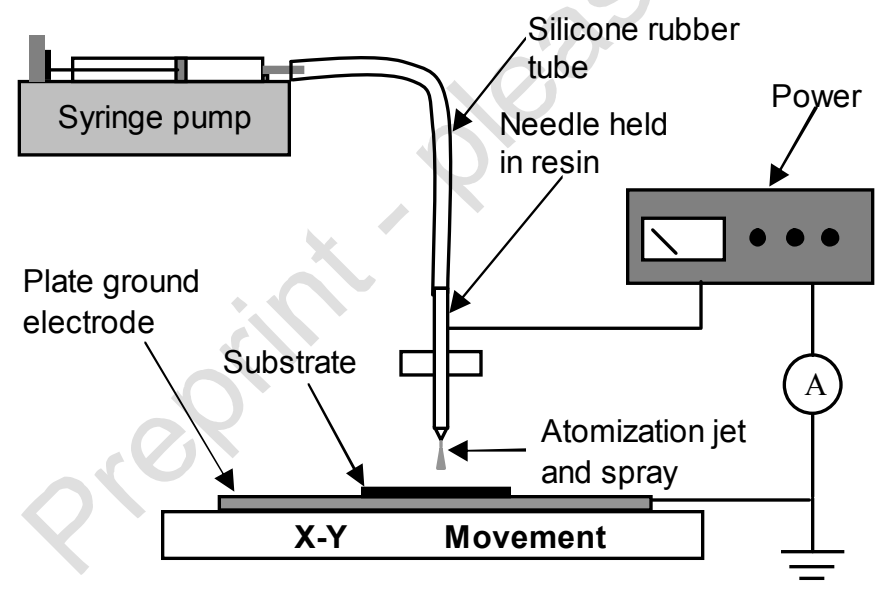

Fig. 2, Schematic representation of EHDA deposition equipment set-up

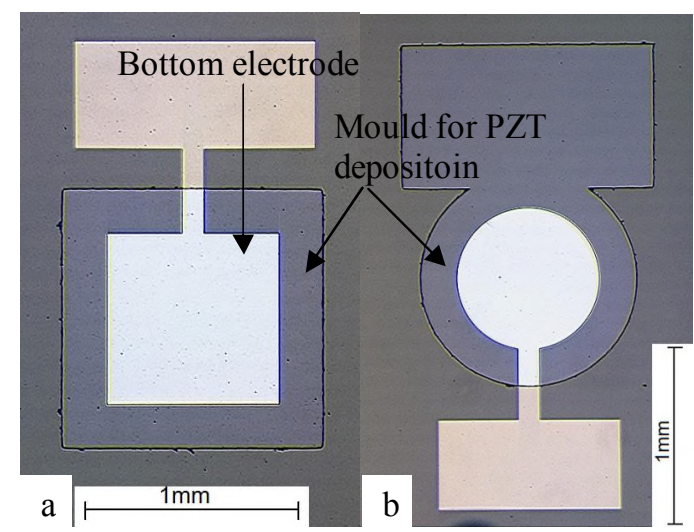

Fig. 3, Optical micrographs showing the photoresist patterned PZT thick film element micromould for EHDA depsotion of PZT structure

The EHDA deposition device is shown in Figure 2, which consists of an electrohydrodynamic needle, a high voltage power supply, a syringe pump and a computer controlled X-Y movement 
stage. The needle has an inner/outer diameter of $0.85 / 1.3 \mathrm{~mm}$ which was connect to a high voltage power supply and a syringe pump. The high voltage power supply (Glassman High Voltage Inc., NJ, USA) was used to apply an electric field between the needle and the ground electrode, and a syringe pump (KD Scientific Inc., MA, USA) was employed to provide the hydrodynamic force to push the PZT slurry up to the outlet of the needle. A thin aluminium plate, serving as the ground electrode, was placed directly on the $\mathrm{X}-\mathrm{Y}$ movement stage and connected to earth potential.

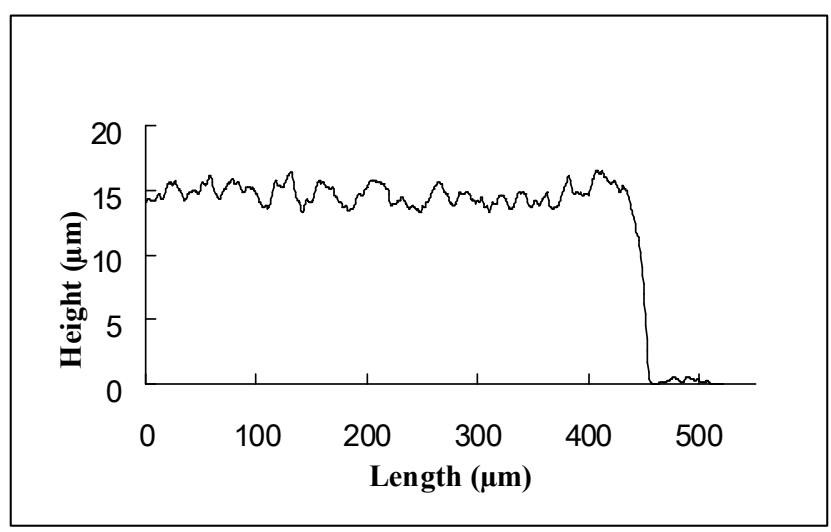

Fig. 4, Surface profile of PZT film element

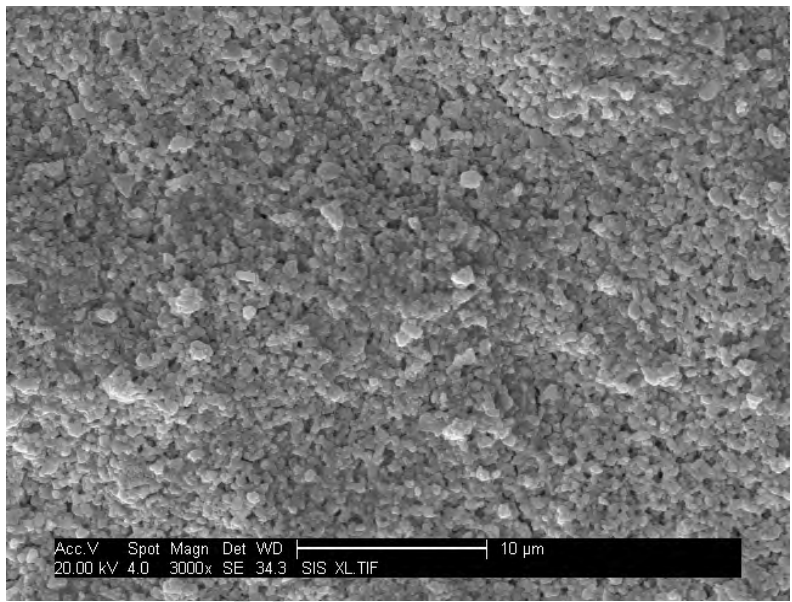

Fig. 5, Scanning electron microscope micrograph showing the surface microstructure of the patterned PZT thick film element after 60 EHDA deposition layers

During the EHDA deposition the patterned photoresist micromould wafer was placed directly on the aluminium plate ground electrode $1 \mathrm{~mm}$ below the needle exit. The PZT film was built up by alternately depositing in the $\mathrm{X}$ and $\mathrm{Y}$ directions on the micromould wafer for 60 layers. After every two intermediate layer depositions (one $\mathrm{X}$ direction scan and one $\mathrm{Y}$ direction scan) the PZT film was dried at $100{ }^{\circ} \mathrm{C}$ for 5 minutes to remove solvent.

After EHDA deposition the structure was sintered at $720^{\circ} \mathrm{C}$ for 20 minutes in a muffle furnace in order to pyrolyze the PZT film and remove the polymeric mould. The final PZT film structure was dipped in acetone for 5 minutes and then cleaned in an ultrasonic bath to remove residual photoresist.

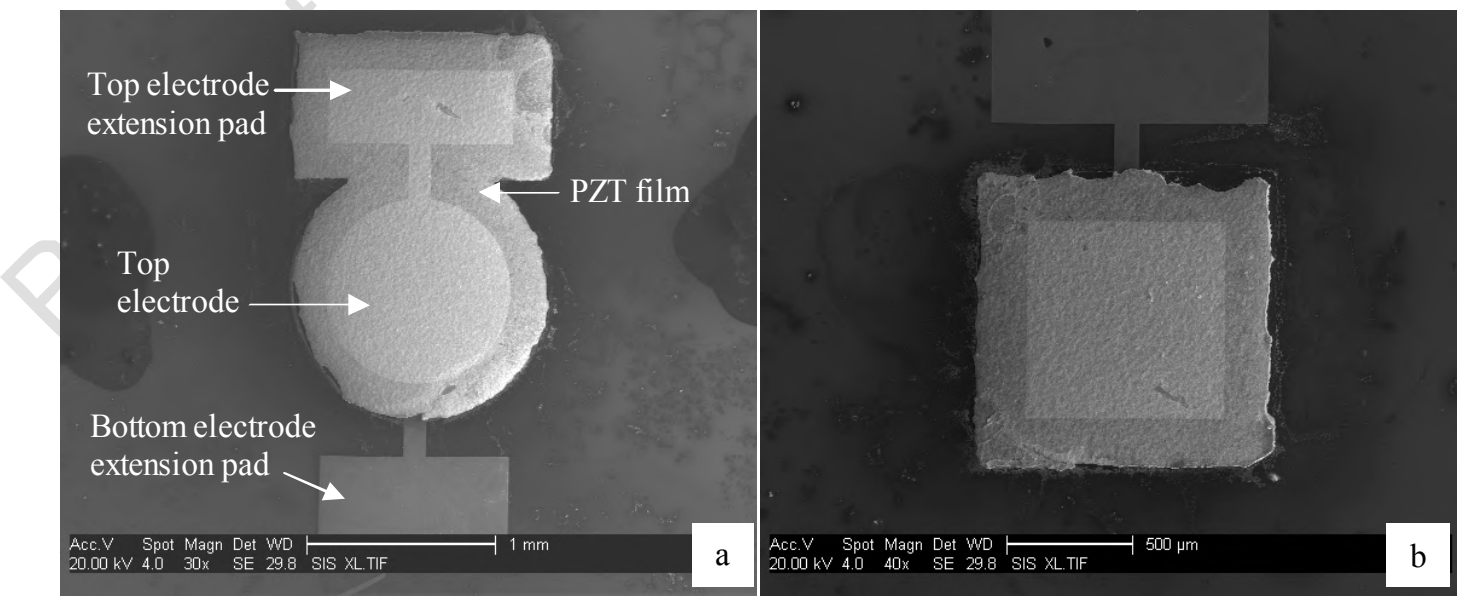

Fig. 6, Scanning electron microscope micrographs showing the patterned PZT thick film single element after 60 EHDA deposition layers: a) circular PZT transducer and b) square PZT transducer 


\section{Results and discussion}

Fig. 3 shows two typical patterned photoresist element moulds (circle and square) for EHDA deposition of PZT film structure, which was $\sim 38 \mu \mathrm{m}$ thick measured by a Surface Profiler (Veeco Instruments Incorporation, UK) and presented uniform dimensional profile. Applied voltage and flow rate are major factors that affect the characteristics of atomization mode and deposition result. In order to obtain a stable jet, the flow rate and the applied voltage were kept constant at $2.2 \times 10^{-10} \mathrm{~m}^{3} \mathrm{~s}^{-1}$ and $5.5 \mathrm{kV}$ during deposition. During the deposition a scan speed of $39 \mathrm{~mm} \mathrm{~s}^{-1}$ and a distance of $4 \mathrm{~mm}$ between two neighbouring parallel paths of deposition were set to ensure a degree of overlap between deposited PZT films. After 60 layers EHDA deposition a $\sim 15 \mu \mathrm{m}$ thick PZT film structure was obtained (Fig. 4).

The patterned PZT element after sintering is shown in Fig. 5. It was observed that the edge of the PZT structure exhibited deformation, which was caused by the deformation and degradation of the photoresist during the solvent removal processes. Heat treatment was needed to remove the solvent in the deposited material to obtain crack-free PZT film structure. Although the $100{ }^{\circ} \mathrm{C}$ used was lower than the AZ9260 photoresist maximum working temperature $110^{\circ} \mathrm{C}$, several heat treatment processes could also cause evaporation of the residual solvent and water and degradation of the photoactive compound [11], which further induced the deformation of the deposited PZT structure. Fig. 6 shows the surface of the patterned PZT element structure after sintering, presenting a well-packed and crack-free film.

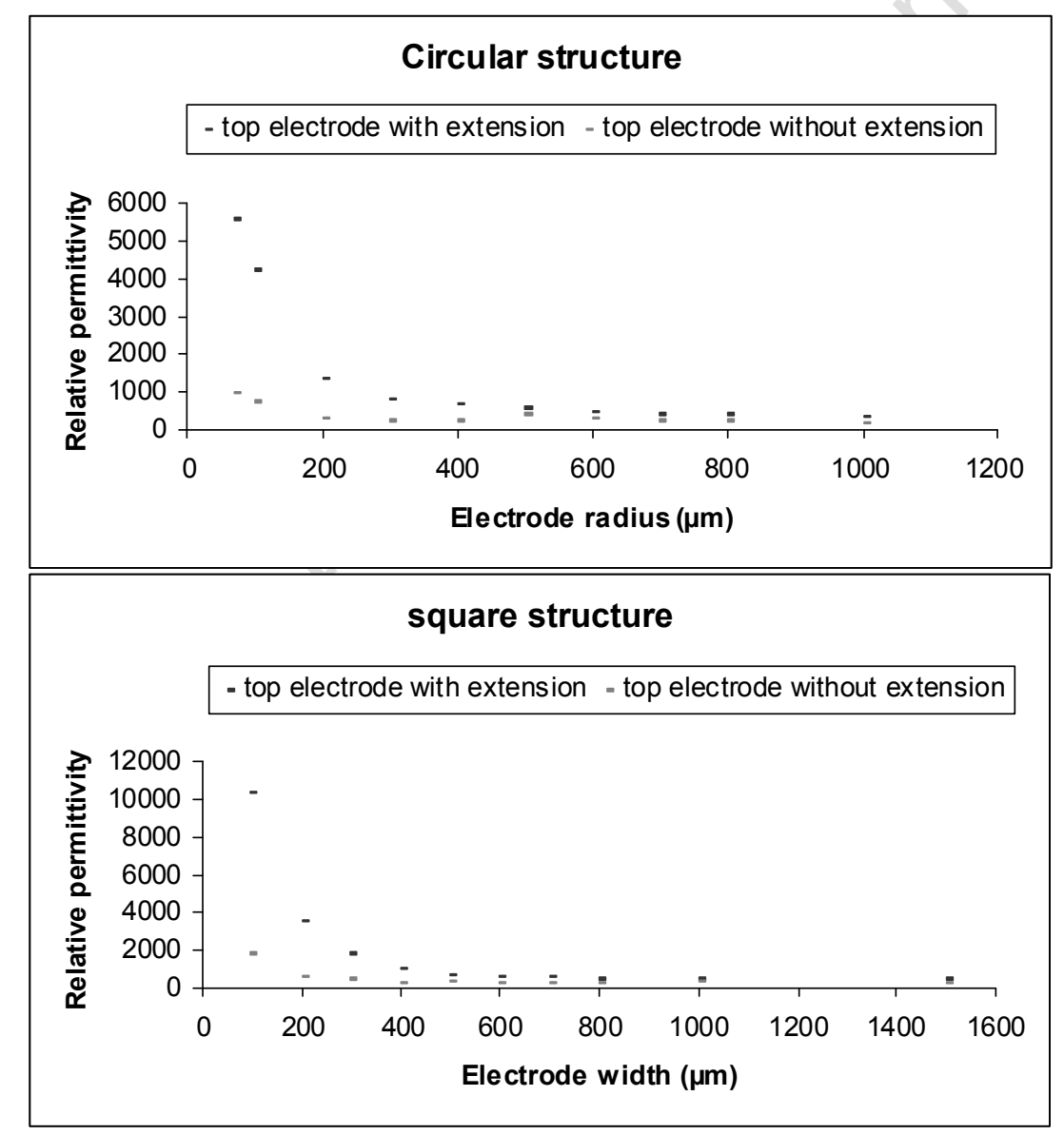

Fig. 7, The relative permittivity of the PZT thick film element with different electrode sizes: a) circular PZT element with various sizes and b) square PZT element with various sizes 
The relative permittivity of the circular and square PZT thick film elements with different electrode sizes is shown in Fig. 7. It was observed that the relative permittivity is much higher with small electrodes (radius or width $<400 \mu \mathrm{m}$ ) than that with bigger electrodes due to the fringe effect [12]. The inter-electrode electric field is not uniform, especially near the electrode edges, where the electric field extends into the space beyond the electrodes, which induces extra relative permittivity. The smaller the electrode size and the bigger the fringe effect. The relative permittivity of these element devices became more consistent and reasonable with electrode radius or width larger than $400 \mu \mathrm{m}$. It is suggested that electrode radius and width of the PZT thick film element bigger than $400 \mu \mathrm{m}$ should be fabricated in order to minimise the fringe effect. It was also observed that the relative permittivity obtained from devices with extension pads was higher than that obtained from devices without extension pads, which was caused by the extension induced extra electric fringing field and capacitance. Attempts were also made to measure the resonant frequency and acoustic response, but were not successful as planned, which was mainly due to the fact that Si back substrate restrained the thickness mode vibration of the PZT film. Further Si back substrate etching process is suggested to solve this problem.

\section{Summary}

This paper demonstrated the use of the EHDA technique combined with polymeric micromoulding for depositing a PZT composite sol-gel slurry to form PZT thick film single element without harsh etching. Circular and square PZT element structures of different sizes were produced. The elements with top electrode extension pad showed higher relative permittivity compared with those without electrode extension. Elements with smaller electrodes (radius or width $<400 \mu \mathrm{m}$ ) had significantly higher relative permittivity than those with bigger electrodes. It is suggested that the radius/width of the single circular/square electrode should bigger than $400 \mu \mathrm{m}$ for $15 \mu \mathrm{m}$ thick elements to reduce the fringe effect and to obtain reasonable relative permittivity. The piezoelectric constant $\left(\mathrm{d}_{33, \mathrm{f}}\right)$ of the PZT element can be consulted from our previous work [13]. Other properties such as resonant frequency and acoustic response of these PZT elements are suggested to be examined after etching Si back substrate. In this work, the use of EHDA deposition and micromoulding technique provides a new, effective and promising manufacturing route for forming piezoelectric microstructures and MEMS devices.

\section{References}

[1] G. H. Haertling: J. Am. Ceram. Soc. Vol. 82 (1999), p. 797

[2] R. A. Dorey and R. W. Whatmore: J. Electroceram Vol. 12 (2004), p. 19

[3] Q. F. Zhou, H. L. W. Chan and C. L. Choy: Thin Solid Films Vol. 375 (2000), p. 95

[4] S. Le Dren, L. Simon, P. Gonnard, M. Troccaz and A. Nicolas: Mater. Res. Bull. Vol. 35 (2000), p. 2037

[5] R. Mass, M. Koch, N. M. White and A. G. R. Evans: Mater. Lett. Vol. 31 (1997), p. 109

[6] A. Jaworek and A. Krupa: J. Aerosol Sci. Vol. 30 (1999), p. 873

[7] M. Cloupeau and B. Prunet-Foch: J. Electrostat. Vol. 25 (1994), p. 1021

[8] Q. Z. Chen, H. B. Zhang, D. Z. Wang, M. J. Edirisinghe and A. R. Boccaccini: J. Am. Ceram. Soc. Vol. 89 (2006), p. 1534

[9] X. Zhao, J. R. G. Evans and M. J. Edirisinghe: J. Am. Ceram. Soc. Vol. 85 (2002), p. 2113

[10] F. Dauchy and R. A. Dorey: In. J. Adv. Manuf. Technol. Vol. 33 (2007), p. 86 
[11] B. Mani: Effect of heat and moisture on thick positive photoresist. In proceedings of the Seventh IEEE/CHMT International Electronic Manufacturing Technology Symposium-Integration of the Manufacturing Flow-from Raw Material through Systems-Level Assembly (New York, 1989, p. 310) [12] G. J. Sloggett, N. G. Barton and S. J. Spencer: J. Phys. A. Math. Gen. Vol. 119 (1986), p. 2725

[13] D. Wang, M.J. Edirisinghe and R.A. Dorey: J. Eur. Ceram. Soc. Vol. 28 (2008), p. 2739 